\title{
A CFD Model of a Headbox for Injecting Dilution Water at Different Locations Along a Step Diffusion Tube
}

\author{
Jinsong Zeng ${ }^{1, *}$, Huang Huang ${ }^{2}$, Yucheng Feng ${ }^{3}$, Ya Dai ${ }^{4}$ and Kuoyan $\mathrm{Ma}^{5}$ \\ ${ }^{1}$ State Key Laboratory of Pulp and Paper, South China University of Technology, Guangzhou, China \\ ${ }^{2}$ State Key Laboratory of Pulp and Paper, South China University of Technology, Guangzhou, China \\ ${ }^{3}$ State Key Laboratory of Pulp and Paper, South China University of Technology, Guangzhou, China \\ ${ }^{4}$ Hongxing Road, No. 86, Sec, Jinjiang district, Chengdu, China \\ ${ }^{5}$ Hongxing Road, No. 86, Sec, Jinjiang district, Chengdu, China \\ ${ }^{*}$ Corresponding author
}

\begin{abstract}
The flow dynamics of the dilution water that adjusts the consistency of the fluid in a hydrodynamic headbox was studied by performing computational fluid dynamics (CFD) simulations. A standard $k-\varepsilon$ turbulence model was used in conjunction with a study of the mass diffusion in the turbulent flow based on the species transport approach. The simulation results show that when dilution water was injected at different sites along the step diffusion tube, the water content at the outlet of the slice channel conformed to a standard normal distribution.
\end{abstract}

Keywords-CFD simulation; hydraulic headbox; consistency distribution

\section{INTRODUCTION}

A headbox is one of the key pieces of equipment used in papermaking machines. Its duty is to keep the pulp suspension uniformly distributed from the outlet of the headbox to the forming wire[1]. To improve the speed and quality of sheet formation, the design of the headbox has progressed from an open-air headbox with agitating rolls to the modern high-speed hydraulic type[2]. The uniformity and quality of a sheet of paper depends largely on the dispersion of fibers at the headbox's discharge points.

In order to design the headbox, full-scale experiments can be expensive; therefore, it would be beneficial to have a computational model that provides information on the effects of various design elements on the flow. Olson developed a theoretical model of the orientations of fibers moving in a turbulent planar contraction and compared the predictions with experimental measurements in the literature[3]. Konstantin built a three-dimensional model to analyze the flow through the headbox, focusing on nonuniformities across the headbox's width and height[4]. Aidun studied the secondary flow in the slice area and Carla A.F. Ventura analyzed the flow distribution in a hydraulic headbox[5]-[6].

To gain a better understanding of the flow field caused by injecting dilution water from different locations in a hydraulic headbox, FLUENT was used to simulate a scale model of an industrial headbox in the paper. In this paper, a standard k- $\varepsilon$ turbulence model with a species transport approach, is used to describe the flow. The results show that at the speed of
$800 \mathrm{~m} / \mathrm{min}$, the hydrodynamic characteristics of the flow field are closely related to the injecting sites, diluted water will affect the consistency, turbulence kinetic energy, and turbulence dissipation rate of the flow channel in the hydrodynamic headbox.

\section{EXPERIMENTAL AND VALIDATION}

A. Experimental

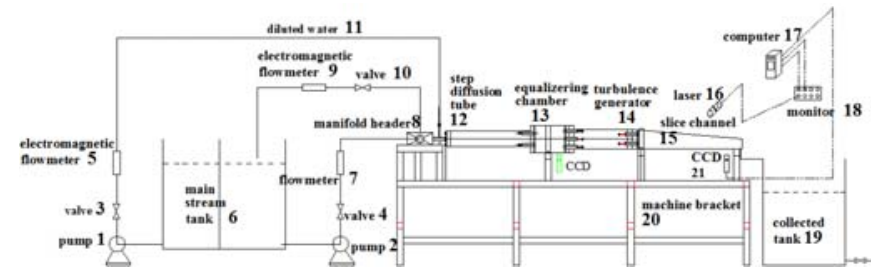

FIGURE I. 1SCHEME OF EXPERIMENTAL SET UP.

To study the scaled industrial headbox model, The experimental set-up is shown in Fig. I; this apparatus consists of a main stream tank made of stainless steel, a scale Plexiglass headbox model, pump 1, pump 2, a polypropylene tank for collecting water 19 , two valves 3 , 4and two electromagnetic flowmeters 5, 9. The scale headbox model is composed of a manifold head 8 , a step diffusion tube 12 , an equalizing chamber 13 , a turbulence generator 14 , and a slice channel 15 . The main stream tank 6 measures approximately $3.0 \times 1.5 \times 2.5 \mathrm{~m}$ with a partition in the middle to transport the different consistency pulp through pump 1 and pump 2. The side of a manifold head with a large and small end is connected to a step diffusion tube 12 . The main stream is pumped to the large end of the manifold head through pump 2. The backflow crossing the manifold head is directly returned to the right portion of the main stream tank when the polyamide particles, used as tracers, are added to measure the flow field. When Rhodamine B (Dantec Dynamics A/S), a fluorescent dye, is added to measure the consistency, the backflow no longer returns to the right portion of the main stream tank 6 and is drained out. The diluted water from the left portion of the main stream tank 6 is pumped into the injector inlet of the manifold head through pump 1. The main stream is mixed with diluted water, passing through the step diffusion tube 12, equalizing chamber 13, 
turbulence generator 14 and slice channel 15 in turn. The mixed flow out of the slice channel is injected into the collection tank. When the flow is used to measure the mixed consistency, it would discharge from the collection tank; when the flow is used to measure the flow field, it would return to the main stream tank to save the tracer and water.

To acquire information about the flow field, a twodimensional PIV (Particle Image Velocimetry) system is combined with a PLIF (Planar Laser Induced Fluorescence) module, which is a non-intrusive optical device (Dantec Dynamics PIV System, Marketec Technology Limited, Hongkong) used to measure the movement of small tracer particles using a CCD (Charge Coupled Device) camera 21, a pulsed laser light 16, a computer 17 and a monitor 18[7]-[9]. $1 \%$ and $0.3 \%$ Rhodamine B solution represent $1 \%$ and $0.3 \%$ pulp suspensions, respectively. The $1 \%$ (by mass) pulp is used as the primary stream, and the $0.3 \%$ pulp is used as the diluted water to be injected.

\section{B. Model and Simulations}

The simulations are performed using ANSYS 12.1. To decrease the computational domain and improve the data describing the other parts of the headbox, except the manifold header, the computational model is as shown in Fig. II, and its sizes are the same as the experiment model, the flow from the seven step diffusion tubes is treated as having equal flux, because the speeds in the manifold header are equal and the step diffusion tubes are the same sizes. Therefore, the computational model includes only the step diffusion tube, the equalizing chamber, the turbulence generator and the slice channel.

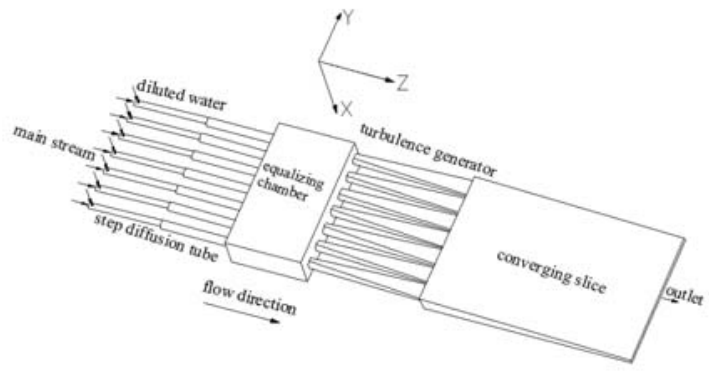

FIGURE II. THE COMPUTATIONAL MODEL.

This computational model includes the following main features: a step diffusion tube, an equalizing chamber, a turbulence generator, and a slice channel. The dilution site is $10 \mathrm{~mm}$ from the inlet of the main stream. The diameter of the dilution water inlet is $8 \mathrm{~mm}$. The small and large pipes of the diffusion tubes are $20 \mathrm{~mm}$ and $30 \mathrm{~mm}$ in diameter, respectively, and $250 \mathrm{~mm}$ long. The inside of the equalizing chamber is approximately $450 \times 250 \times 115 \mathrm{~mm}$. The section of the turbulence generator changes from a circle with a diameter of $35 \mathrm{~mm}$ to a rectangle with dimensions of $55 \times 18 \mathrm{~mm}$; this part is $400 \mathrm{~mm}$ long. The rectangular outlet of the slice channel has dimensions of $470 \times 18 \mathrm{~mm}$ and is $700 \mathrm{~mm}$ long. To clarify the location of the dilution water sites, shown in Fig.III, "4" is the center step diffusion tube, "1" and "7" are the two outside step diffusion tubes, " 2 " and " 6 " are their neighbors, and" 3 " and " 5 " are next to "4". This paper will analyze the dilution water sites combination, where the dilution water is injected from, including "2" and " 6 " combination; " 3 "and" 5 " combination; "4"; "all". "All" refers to that all dilution water sites are injected by dilution water, " 3 "and" 5 " combination refers to that " 3 " and " 5 " dilution water sites are injected by dilution water, and so on.

To model the turbulence, a standard $\mathrm{k}-\varepsilon$ model is combined with a Mass Diffusion in Turbulent Flows Species Transport approach in mixed flow simulations of the viscous model. SIMPLE (Semi-Implicit Method for Pressure Linked Equations) schemes are used for pressure velocity coupling, whereas Standard is used as the spatial discretization method for pressure.

The low consistency pulp flow is considered as a mixture containing two fluids: one is water, the other is the low consistency pulp. Table I listed the simulation parameters for CFD model with the consistency of the diluted pulp is $0.3 \%$, and the main stream is $1 \%$.

TABLE I. SIMULATION PARAMETERS FOR CFD MODEL.

\begin{tabular}{|l|l|l|}
\hline & water & low consistency pulp \\
\hline Density (kg/m3) & 998.2 & 1045 \\
\hline Viscosity (pa $\cdot \mathrm{s})$ & 0.001 & 0.0018 \\
\hline Diffusivity (m2/s) & $2.88 \times 10-5$ & $2.88 \times 10-5$ \\
\hline
\end{tabular}

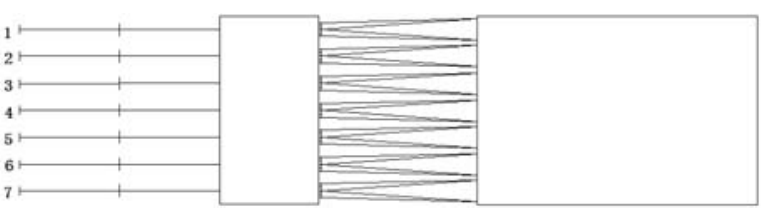

FIGURE III. DILUTION WATER SITES SCHEME.

\section{Model and Simulations Validation}

During the experiment, the pulp consistency is measured. To make the comparison between the CFD predictions and experimental values convenient, the water content is considered as the standard to be compared, the sum of the water content and the pulp consistency are set to be 1. Fig. IV show the results obtained from the PIV experiment and simulation. For Fig. IV(a) and IV(b), the partial flow fields of the equalizing chamber include velocity vectors of $x=0,-0.23 \mathrm{~m}<\mathrm{y}<-0.14 \mathrm{~m}$, $0.12 \mathrm{~m}<\mathrm{z}<0.22 \mathrm{~m}$, and velocity magnitude of the location $\mathrm{x}=0$, $0.23 \mathrm{~m}<\mathrm{y}<-0.14 \mathrm{~m}, \mathrm{z}=0.150 \mathrm{~m}$. In the Fig . IV (a), the left is simulation results, the right is experiment results. It can be clearly seen that the flow fields for the PIV experiments and CFD simulations are the same. 

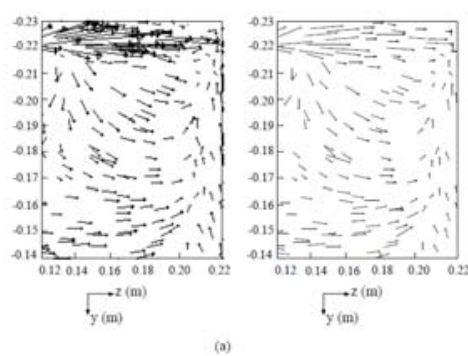

$\Gamma_{y(m)}^{-2(m)}$

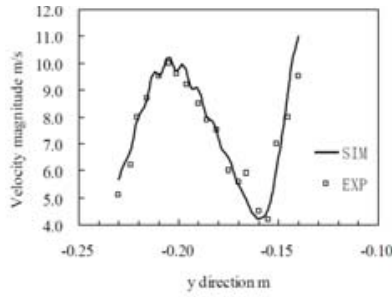

(b)

FIGURE IV. PART EQUALIZING CHAMBER FOR SIMULATION AND PIV AT 800M/MIN. (A) VELOCITY VECTORS, AND (B) VELOCITY MAGNITUDE OF THE LOCATION X $=0,-230 \mathrm{MM}<\mathrm{Y}$ $<-140 \mathrm{MM}, \mathrm{Z}=150 \mathrm{MM}$

\section{RESULTS AND DISCUSSION}

A. Consistency of the Flow at the Slice Outlet of $x=0.030 m$, $z=1.29 m$

As shown in Fig.V, when the main stream is mixed with the dilution water injected at sites"3"and "5", the fluid's water content is highest in the middle of the outlet. As the distance from the center of the outlet increases, the water content decreases. As shown in Fig.VI, when the main stream is mixed with the dilution water injected at site" 4 ", the water content is also highest at the center of the outlet, showing the same tendency as Fig.V.

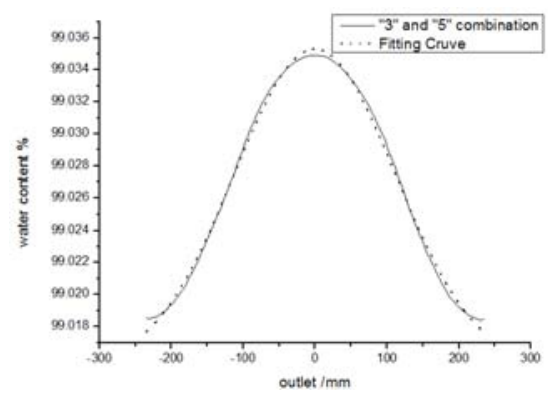

FIGURE V. WATER CONTENT AT OUTLET OF “3”AND "5"COMBINATION.

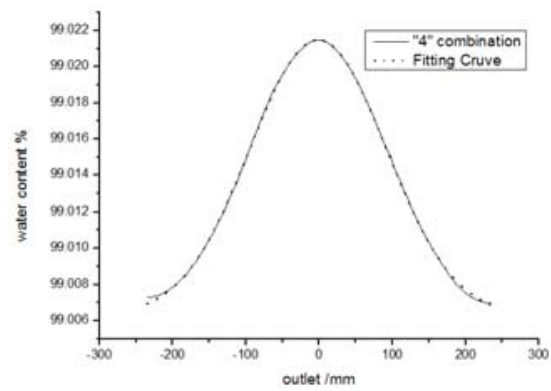

FIGURE VI. WATER CONTENT AT OUTLET OF “3”AND "5"COMBINATION.

To clarify the functional relationship between the location of and water content near the outlet, a distribution equation is deduced by simplifying the theoretical formulae. According to Fig.V and Fig.VI, the water content can be represented by a normal distribution. If we use a Gaussian function to fit the curve, the abscissa value is the length at $x=30 \mathrm{~mm}, \mathrm{z}=1290 \mathrm{~mm}$, $-235 \mathrm{~mm} \leq y \leq 235 \mathrm{~mm}$ and the ordinate value is the water content.

A Gaussian function is as follows:

$$
Y=\frac{A}{\sqrt{2 \pi} \sigma} e^{-\frac{\left(X-X_{0}\right)^{2}}{2 \sigma^{2}}}+Y_{0}(A>0)
$$

As shown in Fig.V and Fig.VI, the fitting coefficient can be greater than 0.99. This shows that the simulation results are in good agreement with the fitting values.

To simplify the function given in equation (1), we set:

$$
\begin{gathered}
\frac{Y-Y_{0}}{A}=\Delta Y \\
X-X_{0}=\Delta X
\end{gathered}
$$

Substituting the above two equations into equation (1) gives the following:

$$
\Delta Y=\frac{A}{\sqrt{2 \pi} \sigma} e^{-\frac{\Delta X^{2}}{2 \sigma^{2}}}
$$

Continuing the analysis of equation (1), $\Delta Y$ is considered the "equivalent consistency" at the outlet of the converging slice. $Y_{0}$ is the offset of lowest point of the curve to abscissa.

Because $\frac{Y-Y_{0}}{A}=\Delta Y, \Delta Y$ has a linear relationship with $Y$. In the expression (1), as $A>0$, it means the curve opening is downward. We can conclude that when water is injected at locations of " 3 " and " 5 " combination, and " 4 " combination, the water content of the fluid at the outlet of the converging slice follows a standard normal distribution.

Next we discuss situations in which dilution water is injected at locations" 2 " and " 6 " combination and at all locations. As shown in Fig.VII and Fig.VIII, at $\mathrm{x}=30 \mathrm{~mm}$ and $\mathrm{z}=1290 \mathrm{~mm}$, i.e., at the outlet of the converging slice, the same location as that represented in Fig.VI and Fig.VII, the main stream is mixed with the dilution water at locations "2" and " 6 "or at all locations, and the water content at the outlet is different at different places. The water content is least at the center of the outlet, which is at $\mathrm{x}=30 \mathrm{~mm}, \mathrm{y}=0 \mathrm{~mm}, \mathrm{z}=1290 \mathrm{~mm}$, and greatest where the diluted water is injected. As shown in Fig.VIII, the water content is least at the center of the outlet, and as the distance from the center increases, the water content also increases on both sides of the outlet.

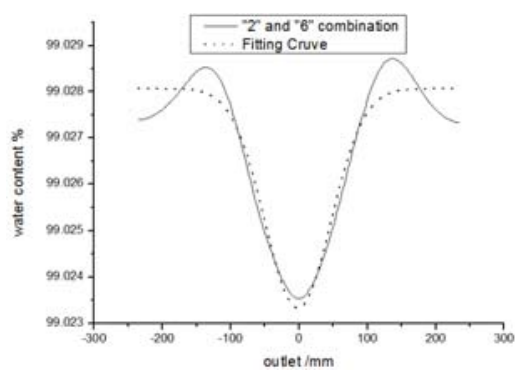

FIGURE VII. WATER CONTENT AT OUTLET OF "2"AND "6"COMBINATION 


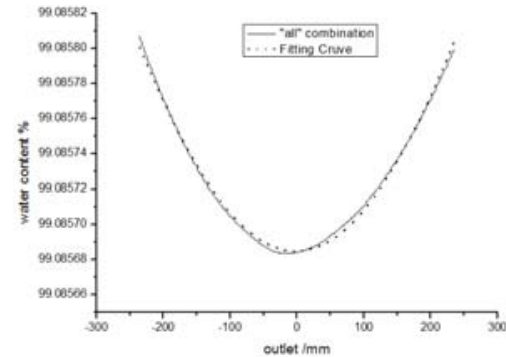

FIGURE VIII. WATER CONTENT AT OUTLET OF "ALL" COMBINATION

To clarify the functional relationship between the location of the outlet and the nearby water content, a distribution equation is deduced by simplifying theoretical formulae. As shown in Fig.VII and Fig.VIII, the water content follows a normal distribution in these cases as well. If we use a Gaussian function to fit the curve, the abscissa value is the length at $\mathrm{x}=30 \mathrm{~mm}, \mathrm{z}=1290 \mathrm{~mm}, \quad-235 \mathrm{~mm} \leq y \leq 235 \mathrm{~mm}$ and the ordinate value is the water content.

A Gaussian function is as follows:

$$
Y=\frac{A}{\sqrt{2 \pi} \sigma} e^{-\frac{\left(X-X_{0}\right)^{2}}{2 \sigma^{2}}}+Y_{0}(A<0)
$$

Using this Gaussian function to fit the curves shown in Fig.VII and Fig.VIII results in a fitting coefficient that is greater than 0.94 for Fig. VII and one that is greater than 0.99 for Fig. VIII.

To simplify equation (3), we set:

$$
\begin{gathered}
\frac{Y-Y_{0}}{A}=\Delta Y \\
X-X_{0}=\Delta X
\end{gathered}
$$

By substituting the above two equations into equation (3), we derive the following formula:

$$
\Delta Y=\frac{A}{\sqrt{2 \pi} \sigma} e^{-\frac{\Delta X^{2}}{2 \sigma^{2}}}
$$

Continuing the analysis of equation (4), $\Delta Y$ is considered the "equivalent consistency" at the outlet of the converging slice. $Y_{0}$ is the offset of lowest point of the curve to abscissa. Because $\frac{Y-Y_{0}}{A}=\Delta Y, \Delta Y$ has a linear relationship with $Y$.

\section{B. Comparison of the Fluid Consistency in Different States}

When dilution water is injected at locations" 2 " "6" combination, the water content at different $\mathrm{z}$ locations after the diluted water has mixed with main stream is as shown in Fig. IX. At $\mathrm{z}=50 \mathrm{~mm}$ in the equalizing chamber, the water content appears peaks at $\mathrm{x}=0 \mathrm{~mm}, \mathrm{y}=-134 \mathrm{~mm}$ and at $\mathrm{x}=0 \mathrm{~mm}$, $y=134 \mathrm{~mm}$, two locations that are just downstream of the sites at which the dilution water is injected. In the equalizing chamber $(\mathrm{z}=50 \mathrm{~mm})$, the water content is higher where the flows from the two injection sites did not completely mix with the main flow. On both sides of equalizing chamber, the water content is the lowest because the injected water has not yet arrived. At $\mathrm{z}=300 \mathrm{~mm}$ (in the turbulence generator) and $\mathrm{z}=1000 \mathrm{~mm}$ (in the converging slice), the flows are more uniformly mixed and the consistency become stable, and the consistency difference decreases.

Accordingly, when dilution water is injected at the locations of " 3 " " 5 "combination and at "all" combinations, the water content at different $\mathrm{z}$ locations after the diluted water has mixed with main stream is as shown in Fig. X and Fig. XI. We reach similar conclusions: at $\mathrm{z}=50 \mathrm{~mm}$ (in the equalizing chamber), the water content appears to have two peaks downstream of injection sites" 3 " "5" combination. These two peaks are closer than the ones downstream of injection sites" 2 " "6" combination. As the flow moves from the step diffusion tube to the converging slice, it became more uniformly mixed. While in the state of "all "combination, there are seven peaks located at downstream of injection sites "all" combination. Similarly, as the mixed stream flow to the turbulence generator and slice channel, water content curve become more and more smooth.

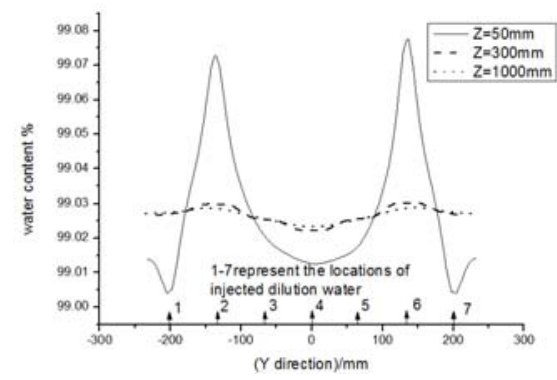

FIGURE IX. WATER CONTENT OF "2" AND " 6 " COMBINATION IN DIFFERENT Z LOCATIONS ( $\mathrm{X}=0$ )

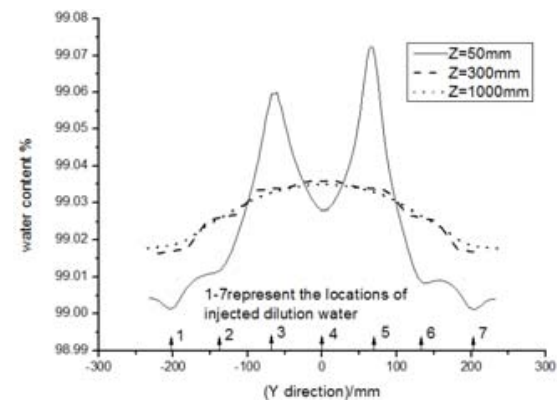

FIGURE X. WATER CONTENT OF “3” AND "5" COMBINATION IN DIFFERENT Z LOCATIONS ( $\mathrm{X}=0$ )

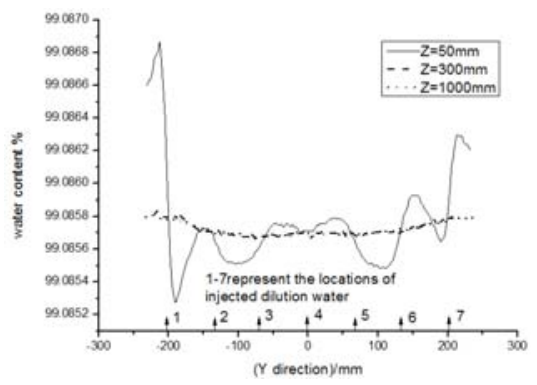

FIGURE XI. WATER CONTENT OF “ALL” COMBINATION IN DIFFERENT Z LOCATIONS ( $\mathrm{X}=0$ ) 


\section{The Turbulent Kinetic Energy and Dissipation Rate at $x=0 m, y=0 m$}

Fig.XII and Fig.XIII illustrate the turbulent kinetic energy and dissipation rate at different $\mathrm{z}$ locations for $\mathrm{x}=0 \mathrm{~m}, \mathrm{y}=0 \mathrm{~m}$ at a flow speed of $800 \mathrm{~m} / \mathrm{min}$. From the expressions of turbulent kinetic energy and turbulent dissipation rate, we can know that they have similar trend. As seen from the figures, when dilution water is injected at location " 4 "or at all of the injection sites, peaks appear near the inlets (at $\mathrm{z}=-0.56 \mathrm{~m}$ ) where the dilution water was injected into the main stream and at the entrance of the turbulence generator at $\mathrm{z}=0.2 \mathrm{~m}$, where the turbulent kinetic energy and dissipation rate were maximized. The main reason for the former situation is that when dilution water is injected into a step diffusion tube from the vertical direction, it causes intensive turbulence and when the mixed flow of the main stream and dilution water arrives at the entrance of the turbulence generator, severe turbulence can appear due to the sharp change of the flow section size. When dilution water is injected at locations" 3 " " 5 "combination or" 2 " "6" combination, no water is injected through the central step diffusion tube; therefore, the turbulent kinetic energy and dissipation rate are lower. Similarly, when the mixed flow consisting of the main stream and dilution water arrives at the entrance of the turbulence generator, the turbulent kinetic energy is maximized, but the turbulent dissipation rate is not as high as it was in the beginning.

To illustrate the turbulent kinetic energy and turbulent dissipation rate in Fig. XII, Fig. XIIIclearly, in the model some critical positions are noted by using the following letters: $a$ : the location where the dilution water is injected; $b$ : the connection between the small and large diffusion tube; c: the entrance of equalizing chamber; $d$ : the entrance of turbulence generator; e: the entrance of slice channel; f: the location $0.1 \mathrm{~m}$ away from the entrance in the slice channel.

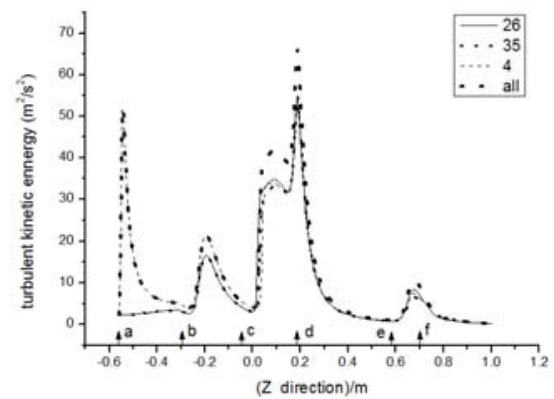

FIGURE XII. TURBULENT KINETIC ENERGY IN Z DIRECTION(X=0M, $\mathrm{Y}=0 \mathrm{M})$

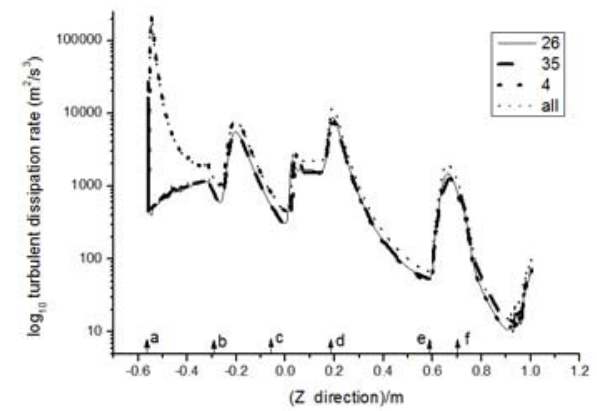

FIGURE XIII. TURBULENT DISSIPATION RATE IN Z DIRECTION $(\mathrm{X}=0 \mathrm{M}, \mathrm{Y}=0 \mathrm{M})$

\section{CONCLUSION}

The flow dynamics of the dilution water that adjusts the consistency of the fluid in a hydrodynamic headbox was studied by using CFD simulations and PIV experiment. The results show that Standard k- $\varepsilon$ turbulence models in conjunction with the mass diffusion in turbulence flow species transport approach can predict CFD model of a headbox for injecting dilution water at different locations along a step diffusion tube; The water content has a standard normal distribution at the outlet of the slice channel; The turbulent kinetic energy and dissipation rate reach the peaks at the downstream sites where the dilution water is injected; Pulp suspension becomes calm and stable as it enters the equalizing chamber, and the equalizing chamber has a role in eliminating and attenuating the energy fluctuations; The dilution water injection locations and the structure of headbox are the two important factors that affect the flow patter of pulp suspension.

\section{ACKNOWLEDGMENT}

The authors acknowledge the financial support of Science and Technology Plan of Guangzhou city (number: No.15020079, Name: Study on Quality Intelligent Control of Modern Paper and Energy-saving Technology with Equipment.)

\section{REFERENCES}

[1] Kefu Chen: Pulp suspensions fluid dynamics in wet end of paper machine, Beijing: Chinese light industry press, 1986.

[2] Kefu Chen: Pulp and Paper Machinery and Equipment, Beijing:Chinese light industry press, 2011.

[3] James A. Olson: Modeling a turbulent fibre suspension flowing in a planar contraction: The one-dimensional headbox, International Journal of Multiphase Flow 30 (2004) 51-66

[4] Konstantin Pougatch: A Computational study of the fluid flow through a hydraulic headbox, Tappi J.,2005.

[5] Aidun, C.K., and Kovacs, A.: Hydrodynamics of the Forming Section: The Origin of on uniform Fiber Orientation, Tappi J., 78 (11), 1995.

[6] Carla A.F. Ventura: Modeling the Turbulent Flow of Pulp Suspensions, Industrial \& Engineering Chemistry Research, 2011.

[7] Jinsong Zeng, Kefu Chen: CFD simulation of dischargers' dilution-zone in high consistency bleaching tower, The Canadian Journal of Chemical Engineering [J], 2011; 476-483

[8] Jinsong Zeng, Kefu Chen:Equal consistency spraying and flow field characteristics of spraying water pipe, The Canadian Journal of Chemical Engineering, 31 DEC 2012

[9] Kefu Chen, Jinsong Zeng: Flow and Simulation of Pulp Fiber Suspensions, Journal of South China University of Technology(Natural Science Edition), Oct 2012 